\title{
Dicerie di un lettore: altre (e inedite) Istruzioni
} perl'uso

Les " rumeurs » d'un lecteur. Modes d'emplois autres (et inédits)

The "Lies" and "Tales" of a Reader. Other (and Unpublished) Directions for

Use

\section{Giulia Cacciatore}

\section{(2) OpenEdition}

\section{Journals}

\section{Edizione digitale}

URL: http://journals.openedition.org/cei/6537

DOI: 10.4000/cei.6537

ISSN: 2260-779X

\section{Editore}

UGA Éditions/Université Grenoble Alpes

\section{Edizione cartacea}

ISBN: 978-2-37747-173-7

ISSN: 1770-9571

Notizia bibliografica digitale

Giulia Cacciatore, "Dicerie di un lettore: altre (e inedite) Istruzioni per l'uso », Cahiers d'études italiennes

[Online], 30 | 2020, Messo online il 01 mars 2020, consultato il 06 mars 2020. URL : http://

journals.openedition.org/cei/6537 ; DOI : https://doi.org/10.4000/cei.6537

Questo documento è stato generato automaticamente il 6 marzo 2020.

(c) ELLUG 


\title{
Dicerie di un lettore: altre (e inedite) Istruzioni per l'uso
}

\author{
Les « rumeurs » d'un lecteur. Modes d'emplois autres (et inédits) \\ The "Lies" and "Tales" of a Reader. Other (and Unpublished) Directions for \\ Use
}

\section{Giulia Cacciatore}

1 Diceria dell'untore arrivò nelle librerie per i tipi di Sellerio nel febbraio del 1981. Contestualmente alla pubblicazione del romanzo, Bufalino fece imprimere dalla tipografia Luxograph di Palermo (la stessa che stampava le opere per Sellerio) l'edizione privata delle Istruzioni per l'uso in 100 esemplari da donare agli amici ${ }^{1}$. Il libretto consta complessivamente di 22 pagine divise in quattro sezioni: Istruzioni per l'uso, Guida-indice dei temi, I personaggi, La scrittura. Si tratta di note autoesegetiche nelle quali lo scrittore raccoglie una parte del materiale accumulato contemporaneamente alla redazione del romanzo, come l'elenco dei personaggi e quello delle citazioni occulte presenti nel tessuto narrativo. Nella Guida Bufalino descrive la natura eterogenea di questo materiale come un «manipolo di notizie, appunti e ipotesi di lavoro, e più o meno false testimonianze, cresciute ai margini del testo, prima, durante e dopo la materiale stesura» ${ }^{2}$.

2 Nel passaggio dall'edizione privata a quella pubblicata con il Club degli Editori nel 1982, Bufalino espunge il paragrafo intitolato Argomento dalla sezione Guida-indice dei temi, e le sezioni I personaggi, La scrittura, mentre amplia la prima parte, Istruzioni per l'uso, aggiungendo quarantaquattro voci di commento, ovvero note esplicative di alcune criptocitazioni presenti nel testo, alle quarantanove dell'edizione privata. La prima edizione tascabile Bompiani delle Istruzioni per l'uso in abbinamento a Diceria dell'untore (1992), invece, recupera la lezione dell'edizione privata integrandola con la versione pubblicata nel 1982. Tra i riferimenti (cripto)citati, che vanno dalla letteratura al cinema, dalla musica all'arte, Bufalino indica, con il relativo numero di pagina del romanzo, Dante, Tasso, Murnau, Watteau, Cellini, Valéry, Proust, solo per fare qualche esempio. 
3 Lo scavo nell'archivio bufaliniano di Comiso consente di affiancare alle notizie d'autore, altre preziose informazioni sulle fonti da lui taciute eppure consultate durante la redazione del romanzo, importanti sia per l'interpretazione complessiva dell'opera d'esordio, sia per seguire le tappe dello studio da lui condotto per la caratterizzazione dei personaggi, per la descrizione dell'ambientazione interna (il sanatorio), ed esterna (il paesaggio, la Sicilia). Queste nuove informazioni sono ricavabili da un foglio di appunti intitolato "Allegazioni Epigrafi e Titoli// Citazioni-Memoranda» ${ }^{3}$. Nel documento Bufalino riporta una lista di autori, di personaggi, o titoli di romanzo, affiancati dal numero di pagina, talvolta da una parola chiave:

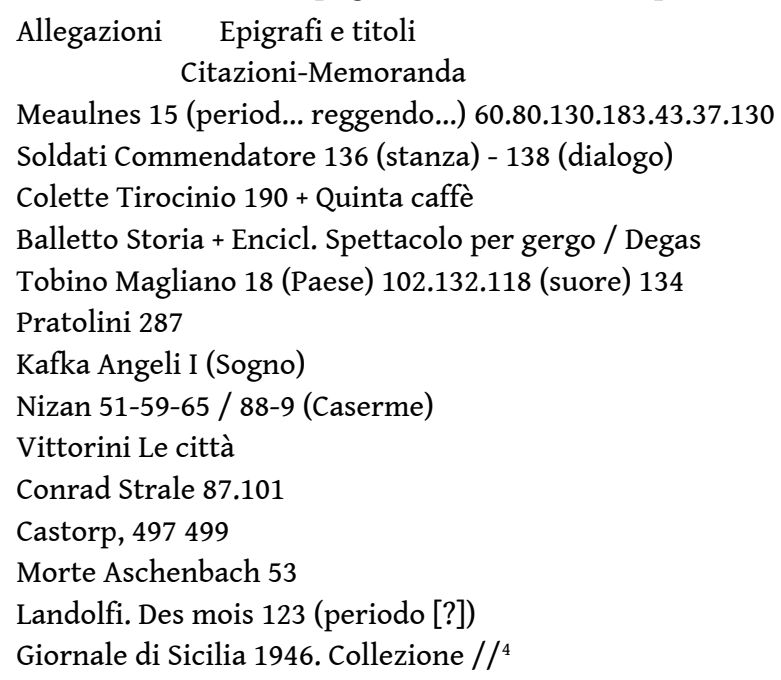

Sebbene la lista non sia esaustiva, come dimostrano gli altri riferimenti indicati dall'autore nelle Istruzioni per l'uso, nonché quelli individuati dalla critica negli studi dedicati al romanzo d'esordio ${ }^{5}$, stupisce la presenza di scrittori italiani mai menzionati da Bufalino, e la cui produzione è coeva alla stesura di Diceria dell'untore, come Soldati, Pratolini, Tobino, Vittorini e Landolfi.

5 Si tratta in tutti i casi di testi appartenenti alla biblioteca personale di Bufalino oggi conservata alla Fondazione di Comiso ${ }^{6}$ : Il grande amico di Alain-Fournier (1913); Il padre degli orfani di Soldati (in A cena con il Commendatore, 1950); Il mio tirocinio di Colette (1924); Le libere donne di Magliano di Tobino (1953); probabilmente La costanza della ragione di Pratolini (1963); Il sogno di Kafka (1914-1915); La cospirazione di Nizan (1938); Le città del mondo di Vittorini (1969); Lo strale d'oro di Conrad (1919); La montagna incantata e La morte a Venezia di Thomas Mann (1924 e 1912); Des mois di Landolfi (1967) ${ }^{7}$. Sebbene la lista faccia pensare inizialmente a delle citazioni poi confluite nel tessuto narrativo di Diceria dell'untore, in realtà si tratta di suggestioni di lettura, spunti da cui trarre materia, una sorta di mappatura dei "memoranda» trascritti durante o subito dopo la stesura del romanzo, nel $1971^{8}$. Il confronto con il testo bufaliniano non permette di stabilire una parentela diretta, di identificare una frase o un rinvio preciso alle pagine appuntate, a eccezione delle opere di Mann e del racconto di Kafka. I libri sono accomunati tra loro dal genere cui appartengono, ovvero quello biografico, o memorialistico, talvolta nella forma del diario come in Des mois di Landolfi. Una lettura attenta delle pagine segnalate nella lista consente di accostare alcuni passi seguendo tematiche specifiche, come la descrizione degli esterni, soprattutto nelle visioni dei paesaggi e del loro mutare con il passare delle stagioni (Tobino, Landolfi); degli interni, evocati nelle ambientazioni bufaliniane del sanatorio, della stanza del protagonista (Alain-Fournier, Nizan); ola caratterizzazione e 
descrizione (psicologica e fisica) dei personaggi (Nizan, Soldati, Conrad, Pratolini, Colette). I rinvii ai romanzi di Mann e al racconto di Kafka, invece, sono funzionali alla descrizione dei paesaggi onirici, alla caratterizzazione dei personaggi e, più in generale, al messaggio complessivo che queste tre opere, soprattutto quelle di Thomas Mann, veicolano.

Bufalino, insomma, inserisce i testi di questa lista in un dialogo ideale, come altrettante coordinate di un sistema letterario cui Diceria dell'untore attinge materia e ispirazione. La modalità di lettura bufaliniana, un metodo di ricerca trasversale che assembla le fonti (distanti e diversissime tra loro) per temi, è dell'antologista più che dello scrittore, e rimanda alle modalità compositive delle antologie inedite allestite selezionando i testi rappresentativi di alcune tematiche come l'amore, l'insonnia, il ricordo ${ }^{9}$. Al fine di ricostruire le modalità di trattamento di questi testi adotteremo lo stesso criterio tematico di Bufalino, riportando gli esempi secondo la funzione che assolvono nel romanzo, trascurando l'ordine della lista e quello cronologico di stampa.

7 Per descrivere la pioggia che prelude alla dimissione del protagonista dal sanatorio, quindi alla sua guarigione intesa come una sorta di nuovo battesimo, Bufalino sembrerebbe fare riferimento a due brani desunti dai romanzi di Tobino e Landolfi. Siamo nel penultimo capitolo di Diceria dell'untore, dopo i funerali di Marta, il protagonista guarda dalla finestra il giardino che circonda la Rocca, imbevuto di pioggia:

Era buio, il giardino, ma distinsi il lustrare di una cesoia dimenticata nell'erba, percepii la soddisfazione delle radici dentro la terra bruna e bagnata. È piovuto, ecco dunque l'autunno. [...] Intanto quieta quieta veniva giù di nuovo la pioggia. Io restavo col capo sporto fuori a metà, sotto l'acqua che gocciava dai coppi del tetto, $\mathrm{e}$ mi sentivo stranamente lieto. o pago, piuttosto, mentre guardavo nel giardino il prato imbeversi ancora e l'acqua battere il suo mite alfabeto sulle sedie di ferro rovesce, sul fogliame e gli aghi degli alberi. Bisogna che parta, mi dissi [.... ${ }^{10}$.

8 La scena evoca due luoghi delle Libere donne di Magliano di Tobino e di Des mois di Landolfi. Nel primo libro, in cui l'io narrante coincide con lo psichiatra che appunta sul suo quaderno gli studi condotti sui pazienti, la narrazione indugia su una brevissima descrizione del manicomio di Magliano, che richiama le alte mura antistanti la Rocca bufaliniana, nonché la focalizzazione interna della narrazione, lo sguardo sereno del protagonista che contempla il giardino bagnato dalla pioggia:

[...] il manicomio, che possiede intorno una bella campagna e giardini tra le sue mura, è tutto gocciolante d'acqua piovana ché son circa venti giorni, ed è maggio, che ogni poche ore il cielo nuvoloso scrolla una grondaia; le erbe verdissime e pulite sono cresciute per ogni dove con grandi baffi; [...] un felpato sereno ricopre e offusca tutta la cerchia del manicomio; i malati, essendo chiusi a causa del tempo nelle sale di soggiorno, non si sentono; c’è quella sonnolenta pace come si fosse d'autunno quando nelle cantine gorgoglia lentamente il mosto ${ }^{11}$.

9 Anche la pagina appuntata da Bufalino di Des mois di Landolfi descrive la pioggia, stavolta in chiave di una vera e propria personificazione, come una 'voce' che incita il protagonista a lasciare il sanatorio, in particolare nella chiusa del brano già citato, «Bisogna che parta, mi dissi»:

La pioggia era una sfida e un risucchio; come in certi miti, quando i campioni cadono dal cielo, ciascuna gocciola battendo il suolo si trasformava in cavaliere armato; di tra le cortine d'acqua partiva un richiamo; l'aria tutta, misteriosamente rinnovata, m'intronava gli orecchi: «Vieni. Tu dicevi di voler fare qualcosa: questo è il momento. E non qualcosa, tutto potrai fare» ${ }^{12}$. 
La descrizione della Rocca in estate, l'azzurro del cielo che sovrasta il sanatorio ${ }^{13}$, sembra trovare il suo corrispettivo in un altro luogo del romanzo di Tobino, alla pagina 134, e in particolare nelle caratteristiche con cui viene rappresentato il manicomio di Magliano nella stessa stagione ${ }^{14}$.

11 Quanto agli ambienti interni, la sequenza in cui è descritto il capannone dove il protagonista di Diceria sospetta che Marta e il Magro abbiano consumato un rapporto amoroso, sembra richiamare il romanzo Il grande amico di Alain-Fournier:

[...] m'abbandonai contro la porta. Fu Sebastiano ad accorgersi che cedeva sotto la spinta dei dorsi, era chiusa solo a metà. Non restò dunque che entrare [...] un giaciglio a terra c'insospettì, gualcito un poco da una pressura recente di membra, e inoltre sparso di mucillagini e capelli, come un letto di nozze abbandonato all'alba [...] Sebastiano, fu peggio. La faccia gli si cangiò in una maschera losca, come di un bambino che piangerà fra un istante ${ }^{15}$.

Alla pagina 60 del Grande amico, infatti, si legge:

Meaulnes s'avvicinò. Doveva trattarsi di una specie di grande parco per il bestiame, o di un ovile abbandonato. La porta cedette con un gemito. La luce della luna, quando il gran vento metteva in fuga le nubi, filtrava attraverso le fessure dell'assito. Vi regnava un odore di muffa. Senza cercare altro, Meaulnes si distese sulla paglia umida, con i gomiti in terra e la testa tra le mani [...]. Pensò allora alla coperta della giumenta che aveva lasciato sulla strada e si sentì così infelice, così adirato contro se stesso che gli venne una gran voglia di piangere ${ }^{16}$.

13 A rafforzare la contiguità testuale tra le due opere è anche la corrispondenza tra il rapporto d'amicizia intrattenuto dall'io narrante di Diceria con Sebastiano, e quello di François Seurel, io narrante del Grande amico, con Meaulnes, il protagonista. Il racconto della vicenda di Sebastiano prima del sanatorio, il periodo della malattia e il triste epilogo, il disperato gesto del suicidio nella tromba delle scale, infatti, è affidato all'io narrante di Diceria, che precisa sin dalle prime pagine del romanzo come l'amico morto fosse «nel suo albo di croci, quella che tuttora fa male» ${ }^{17}$. Sebastiano, pertanto, è una figura centrale nella ristretta cerchia degli affetti del protagonista nel microcosmo del sanatorio, e il loro rapporto, costruito più sul non detto che su dialoghi espliciti, ricorda in parte quello intrattenuto tra François e Meaulnes. Questa ipotesi giustificherebbe la presenza di un nutrito numero di luoghi del Grande amico appuntati da Bufalino nella lista e, al contempo, l'assenza di riprese dirette dal testo di AlainFournier.

La caserma descritta nel romanzo La cospirazione di Nizan, invece, in cui Simon meditava la fuga da Parigi, invogliato dal fischio dei treni udito in lontananza ${ }^{18}$, richiama sia la stanza di Marta che la stessa smania di evasione provata nel silenzio della notte e alimentata dal suono dei treni in corsa ${ }^{19}$.

Più interessante è l'uso che Bufalino fa delle fonti per la caratterizzazione dei suoi personaggi. Anche in questo caso non siamo in presenza di riprese dirette dai libri riportati nella lista. Gli eroi dei romanzi cui lo scrittore si ispira 'prestano' le loro caratteristiche e la loro storia personale a quelli di Diceria. È come se Bufalino avesse cercato di conferire loro un passato ideale, letterario. Lo scrittore non ricorre a fonti storiche per ricostruire gli avvenimenti da porre sullo sfondo del romanzo, bensì si affida a suggestioni letterarie che, seppur fittizie, sono in grado di restituire $i$ sentimenti, le impressioni legate alle vicende dei suoi personaggi. Gli esempi sarebbero molti, citeremo solo quelli che ci paiono evidenziare un legame diretto con il testo bufaliniano. 
16 I protagonisti della Cospirazione di Nizan, giovani studenti con ambizioni rivoluzionarie poi fallite, vivono il presente senza pensare alle conseguenze delle loro (potenziali) azioni sovversive, senza sentirne il peso, come gli stessi giovani reduci di Diceria dell'untore, che mentre partecipavano alla guerra, non percepivano del tutto (come avrebbero fatto più tardi, negli anni immediatamente successivi) la gravità dell'uccidere il nemico, la follia di agire contro i loro principi per rispettare gli ordini di un superiore, ma erano in preda alla spensieratezza giovanile:

Di tanti giorni di ebrezza e di felici rincorse sugli Appennini, coi fazzoletti di colore attorno al collo e i soprannomi da romanzo, restavano a galleggiare pochi scarabocchi e stemmi di atrocità o amore: un fischio d'intesa, un fumo di sera su una cascina, un crepito di parabellum davanti a noi, in un sentiero senza scampo. Mentre, sopra ogni altra cosa, soverchiando ogni lutto o splendore della memoria, ferinamente trionfava nel vento il tanfo della città bombardata ${ }^{20}$.

17 Il protagonista di Diceria dell'untore rievoca, a distanza di venticinque anni, con il distacco di un reduce ormai maturo e consapevole, le vicende della guerra e della tubercolosi, proprio come le esperienze di Laforgue e dei suoi compagni (avvenute negli anni '20 del Novecento) sono raccontate successivamente, quando la distanza dagli accadimenti consente al narratore di ricostruirne più chiaramente le cause e gli effetti, o di enfatizzare l'inconsistenza dei loro propositi:

[...] nulla è più difficile dello sfruttamento metodico di un avvenimento del cuore, nulla si acquieta più rapidamente delle onde di un colpo di fulmine. Gli esami, la pigrizia, la letteratura, la curiosità per la donna, tutti i falsi maneggi in cui si disperde la vita degli adolescenti impedirono per lungo tempo a Laforgue e ai suoi amici di trarre dai ricordi del 24 e 25 novembre tutte le conseguenze pratiche che avrebbero dovuto implicare; la cosa per anni e anni fece parte solamente dei loro ricordi ${ }^{21}$.

18 La distanza tra il concetto di felicità maturato dai personaggi di Diceria prima della guerra e quello successivo agli eventi bellici è anche al centro del dialogo contenuto nel racconto Il padre degli orfani di Soldati - di cui Bufalino appunta la pagina facendola seguire dall'indicazione «dialogo» - in cui il commendatore spiega la sua concezione di «coscienza» e di «verità»:

«[...] come vedi io sono tranquillo. Non sono mai stato così felice. Credo di avere fatto a puntino quello che era il mio dovere».

«Beato te» sospirai.

«Perché? Non mi credi?» fece brusco, irritato. [...] «Perché, per esempio, ricordo benissimo che anche prima, le rare volte che ti sei lasciato andare con me a questi discorsi (e ciò accadde molti anni or sono) mi dicesti appunto la stessa cosa [...] siccome hai sentito il bisogno di cambiar vita, o non eri sincero allora, o non lo sei oggi. Voglio dire, sincero con te stesso».

«No, caro» rispose dopo una breve pausa e con troppa calma, «era, ed è, la verità. Soltanto che la verità cambia. È cambiata, questi anni, con la guerra, non soltanto nelle nazioni e nelle società, ma anche nella coscienza delle persone».

«Avevo sempre pensato che tu non ti preoccupassi della tua coscienza».

Anche questa volta tardò un attimo a rispondere: «Può essere vero. Sì, può essere stato vero; ma appunto, fino alla guerra $[. ..] »^{22}$.

19 Il riferimento al racconto Lo strale d'oro di Conrad sembra rinviare a una (possibile) sintesi dei significati più profondi di Diceria dell'untore, poiché vi si legge in filigrana la malinconia e la tristezza del protagonista per la perdita dei compagni del sanatorio, il ricorso alla memoria (e alla fantasia) per ricomporre l'immagine di quelle persone, 
l'importanza del recupero del passato per lasciare traccia di quei giorni altrimenti perduti nell'oblio del tempo:

[...] ma egli doveva già essere lontano; e devo aggiungere ch'io non lo vidi mai più in vita mia. Il suo apparire nelle mie visioni d'allora, fu come quello di altre figure di quel tempo: indimenticabile, un po' fantastico, utile per chiarire le mie osservazioni, per illuminare i miei ricordi che ancora oggi combattono quelle chiare luci e le orribili ombre di quei giorni indimenticabili ${ }^{23}$.

20 Altre fonti, invece, sono direttamente riconducibili alla caratterizzazione del protagonista di Diceria, come se Bufalino avesse cercato di conferirgli spessore letterario traendo spunto dai suoi antesignani. È il caso (nuovamente) dell'io narrante delle Libere donne di Magliano che trascrive le riflessioni scaturite dalla sua esperienza di 'clausura' nel manicomio accanto alle donne che egli, con dedizione, assiste. Questa sorta di «claustrofilia», come la chiamerebbe Bufalino, è ben espressa in una delle pagine da lui appuntate nella lista, e rinvia ai sentimenti provati dal protagonista in attesa della morte, durante il lungo ricovero nel sanatorio:

La mia vita è qui, nel manicomio di Lucca. Qui si snodano i miei sentimenti. Qui sincero mi manifesto. Qui vedo alberi, tramonti, il tempo scorre nella mia attenzione. Dentro una stanza del manicomio studio gli uomini e li amo. Qui attendo: gloria e morte. Di qui parto per le vacanze. Qui, fino a questo momento, sono ritornato ${ }^{24}$.

21 Nel testo di Tobino ritroviamo anche altri passi, segnalati da Bufalino nella lista, in cui vengono descritti i personaggi femminili, alcuni dei quali affetti dalla tubercolosi:

La Sbisà ha nel naso la tubercolosi; s'indovina che c'è un roditore in mezzo alla sua faccia. È veneta, pallida, buona. La pazzia la sospinge, contro la sua volontà, a delirare. Stamani diceva con disperazione «Le parole mi corrono, debbo discorrere, non posso, non posso più».

Era chiaro che desiderava in qualche modo una soluzione, cioè la pace, che può essere anche la morte ${ }^{25}$.

E stamani Carlino, il tecnico di laboratorio, mi ha detto che il Bedetti da dieci giorni è al sanatorio perché tubercoloso e una forma aperta, già avanti e distesa.

M'è venuto uno stupore doloroso: è probabile l'abbia presa dai malati perché a volte qui un tubercoloso, specie se agitato, ne infetta cento prima che lo si scopra ${ }^{26}$.

Quanto al tormentato rapporto sentimentale con la ballerina Marta, il suo 'precedente letterario' potrebbe essere il triangolo amoroso tra i protagonisti del romanzo di Pratolini La costanza della ragione: il condizionale in questo caso è d'obbligo poiché nella lista Bufalino trascrive soltanto il nome dell'autore seguito dal numero di pagina (287). Tuttavia, confrontando tra loro i testi pratoliniani posseduti da Bufalino nella sua biblioteca, questo sembra essere l'unico libro ad avere dei rapporti diretti con Diceria dell'untore per la materia narrata, e per la presenza di alcune note di lettura (una piega sul margine superiore alle pagine 19 e 22) che sembrano avvalorare la nostra ipotesi. Un ulteriore elemento di parentela tra le due opere è la citazione della Vita Nova posta in esergo alla Costanza della ragione, opera dantesca cui Bufalino dichiarò più volte di essersi ispirato per il romanzo. Il triangolo amoroso tra Bruno, la ragazza da lui amata Lori, e Luigi, al centro della Costanza della ragione, rinvierebbe a quello tra il protagonista di Diceria dell'untore, Marta e il Gran Magro. In particolar modo, sembra esserci una relazione diretta tra le impressioni che prova Bruno alla vista dell'agonia di Lori, che ha contratto una meningite inguaribile, e quelle del personaggio principale di Diceria, che vede nella fine di Marta un sacrificio, anticipato dal simbolico «sacrificio della Donna» nella partita a scacchi con il Gran Magro, necessario alla sua sopravvivenza il cui scopo è quello di testimone della loro avventura. Il protagonista, 
tuttavia, pur essendo scampato alla morte, non riesce a liberarsi dal senso di colpa provato nei confronti degli amici morti nel sanatorio, e in particolare per la donna, come dichiara anni dopo: « [...] è maggiore il rimorso che non il sollievo, d'aver tradito a loro insaputa il silenzioso patto di non sopravviverci». La sua sopravvivenza viene pertanto interpretata come l'esaudirsi di una volontà, dichiarata già nelle prime pagine del romanzo, «Era divenuto un gioco alla Rocca volere o disvolere morire»:

[...] la sua debolezza e la mia codardia la uccidevano, come nel male che si era impossessato del suo corpo e altrettanto schifosamente lo deturpava [...]. Ci si perde quando si decide di perdersi, ora mi dicevo. E mi sentivo da me stesso e da tutti tradito [...]. Era davvero un gioco che c'inventavamo la vita, quando dicevamo di essere senza passato, concreti nella consumazione del nostro amore, noi stessi garanti del suo avvenire? [...]. Ella si era perduta per salvare il nostro amore, dividendosi tra me e lui; e nella caligine dell'agonia, ci aveva identificati [...]. Una povera avventura la nostra, simile a quella di coloro che ci circondavano, ripetibile all'infinito [...]. Io sarei rimasto, perseguitato dal suo ricordo, colpevole della mia ignavia, ma per sempre disincantato, pronto d'ora in avanti ad intravedere il male nelle sue più perfette mascherature, e ad affrontarlo con astuzia. A ricambiarlo con ferocia ${ }^{27}$.

In questo contesto si inserirebbe anche il riferimento nella lista alla morte di Gustav von Aschenbach, il protagonista della Morte a Venezia di Mann. Nel quinto capitolo ${ }^{28}$, l'ultimo del romanzo, sono presenti alcuni elementi che potrebbero essere stati ripresi da Bufalino in Diceria dell'untore: la corrispondenza tra il colera che infierisce su Venezia e la malattia dello scrittore tedesco; lo stato di decadimento in cui versa la città in seguito alle menzognere dichiarazioni delle Autorità, che negano la presenza del morbo nel territorio della Serenissima, metafora della degenerazione morale dello scrittore sempre più coinvolto dalla passione segreta per il giovane polacco Tadzio. Le stesse corrispondenze si trovano nella Palermo squassata dalle bombe che fa da scenario al romanzo di Bufalino e in particolare agli appuntamenti tra il protagonista e Marta ${ }^{29}$. Sin dal primo incontro, i due sono come assorbiti dalla città in sfacelo, derisi dalla popolazione del quartiere della Kalsa (uno dei più colpiti dalle bombe sganciate su Palermo dagli angloamericani nel '43) per le loro effusioni accanto al distrutto Palazzo Sclàfani che ospitava l'affresco Il trionfo della morte, simbolico ma chiaro riferimento al loro comune destino. La 'corruzione' di Marta, il suo passato di kapò, è personificata dalla città che accoglie i due ammalati, una sorta di correlativo oggettivo della compromissione della donna. L'appunto di Bufalino che si riferisce alla «morte di Aschenbach", sarebbe dunque da mettere in relazione con il personaggio femminile e il suo destino, non con la vicenda del protagonista che fa pensare piuttosto al giovane, ingenuo, Tadzio. L'avanzare della malattia in Aschenbach e il suo appressarsi alla morte è scandito da avvenimenti come il concerto cui assistono, separati, Gustav e Tadzio. In modo simile, in Diceria dell'untore, Marta e il protagonista assistono, prima della morte di lei, alla tragedia eschilea dell'Agamennone re e, sebbene uniti, la loro distanza è segnata dal differente destino che li aspetta (la morte di lei, la salvezza di lui). Il sogno premonitore che annuncia la morte a Gustav rimanda al sogno che il protagonista di Diceria fa due volte, all'inizio del romanzo e poco prima della morte di Marta, in cui capisce che la donna è prossima alla fine. Ultimo elemento comune ai due testi è l'«atmosfera autunnale» che fa da sfondo alla scena della morte di Gustav: come il protagonista guarda Marta morire, con il distacco di chi osserva senza intervenire, così Tadzio assiste da lontano alla morte dello scrittore e gli fa un cenno con la mano, come a indicargli una prospettiva lontana di sublimazione della morte e della malattia, una 
visione del corpo che si libera dalle sofferenze. In questo caso il riferimento presente nella lista sembra dunque rinviare a una parentela più diretta tra le due opere rispetto agli altri testi presi in esame, poiché Bufalino riprende dalla novella di Thomas Mann tutti gli elementi (lo spettacolo, il sogno, l'atmosfera autunnale) che precedono la morte di Marta.

Riguardo la caratterizzazione del personaggio femminile, la descrizione fisica di Viola nelle Libere donne di Magliano ${ }^{30}$ fa pensare a quella di Marta, alla sua agilità («guizzava nel cielo») e alla sua magrezza («membra minute») che le consentono di concludere il difficile numero di danza, voluto dal Magro per lo spettacolo annuale organizzato nel sanatorio, nonostante il respiro spezzato dalla tubercolosi. La sua esile figura doveva evocare quella delle ballerine di Degas, unico artista presente nella lista di riferimenti stilata da Bufalino.

La minuziosa descrizione degli abiti del personaggio di Camille nel Grano in erba ${ }^{31}$ di Colette, che a sua volta troviamo in una delle pagine appuntate da Bufalino nella lista, può averlo ispirato per la raffigurazione delle vesti di Marta, di cui specifica i colori, $i$ tessuti, l'effetto di eleganza che conferiscono al portamento della donna ${ }^{32}$.

Infine, per quanto pertiene alla visione onirica del personaggio di Marta, Bufalino può avere attinto nuovamente al romanzo di Tobino. In un passaggio lo psichiatra del manicomio di Magliano spiega la dissociazione mentale di una paziente ricorrendo all'immagine di una donna seduta in una gondola che sporge le mani verso gli spettatori, i quali la vedono distintamente e la odono mentre dice con voce limpidissima ma disumana: "voglio andare a casa...». La donna «scorre all'infinito» nella gondola «e voi, poiché la vedete da un altro mondo, che essa non incontrerà mai, la comprendete come un chiaro assurdo e notate dunque che non ha più leggi umane, cioè con le vostre leggi è incomprensibile» ${ }^{33}$. Questo brano rinvia alla descrizione di Marta vista in sogno dal protagonista, quindi in uno stato di incoscienza, in cui il «chiaro assurdo» sta nella visione ultraterrena della donna già morta, annegata, e non di una persona viva:

Allora una bagnante entrava nel mio sognare, con un costume tutto nero e una gocciola di mare sulla coscia sporca di sabbia, e oscillava su e giù nell'aria, come da un'amaca di nebbia che dondolasse sul mio capo adagio, su e giù sempre, per l'eternità. Il ventilatore parlava da un angolo della stanza, e le scompigliava i capelli. Ed erano i capelli di Marta, i capelli di un'annegata ${ }^{34}$.

Un discorso più approfondito merita il confronto tra la componente onirica presente nel romanzo bufaliniano e i testi citati nella lista. La morte a Venezia è uno dei tre modelli ripresi per la costruzione del sogno, insieme all'altro capolavoro di Mann, La montagna incantata, e al racconto di Franz Kafka Il sogno. Da un confronto tra questi tre testi, e in particolar modo tra i capitoli cui appartengono le pagine indicate da Bufalino, si evince una differente modalità nel trattamento dei brani rispetto agli esempi precedentemente evocati: in questo caso infatti nel tessuto narrativo bufaliniano $i$ riferimenti al 'testo di base' sono più puntuali e diretti, sebbene effusi e rielaborati in uno stile diverso.

Per la costruzione del sogno in apertura del romanzo Bufalino mescola alcuni elementi della sezione intitolata Neve della Montagna incantata con il racconto Il sogno di Kafka. Diceria dell'untore prende avvio dal sogno ricorrente del protagonista ${ }^{35}$ che, passando di "grotta in grotta», avvolto da un'atmosfera lugubre e spinto dal vento, raggiunge una scarpata. Calato il vento («il silenzio era pieno»), lentamente egli discende e, arrivato 
nella profondità della scarpata, vede degli uomini seduti, vestiti di impermeabili, che pronunciano parole incomprensibili. Lui è in ginocchio, con le mani dietro la schiena, $\mathrm{e}$ guarda il più anziano di questi uomini che, curvandosi un momento, disvela la presenza di una donna, («la dissepolta e rapida nuca di lei») Marta. Il sonno non è pieno, bensì una sorta di dormiveglia («uno stato di sdoppiata vitalità») dal quale, tuttavia, egli riesce a svegliarsi a fatica. Le simmetrie con la sezione Neve del sesto capitolo della Montagna incantata - di cui Bufalino appunta nella lista le due pagine che vanno da 497 a 499 - sono molteplici: Hans Castorp, nonostante le raccomandazioni del medico curante, compra gli sci e si avventura sulla montagna, le cui vie erano «fiancheggiate da pareti di neve superanti l'altezza di un uomo e simili a superfici alabastrine». Accompagnato da un profondo silenzio («il silenzio intorno era inesorabilmente vuoto») e dalla clemenza del clima, Castorp procede verso le alture; ben presto, tuttavia, sopraggiunge una tormenta che, sebbene prevedibile, non gli impedisce di allontanarsi ulteriormente dall'abitato, ubbidendo a una sorta di inspiegabile provocazione nei confronti della natura, di attrazione per il pericolo, una «tentazione di abbandonarsi $\aleph^{36}$. Dopo aver girato a vuoto sospinto dal vento, Castorp trova momentaneo rifugio dietro una capanna dove, appoggiato al muro, cede al sonno. Immagina di essere ai piedi di un monte che guarda verso il mare, un mare del sud («Napoli o la Sicilia») pur non essendovi mai stato, e di osservare i bagnanti divertiti. A questo paesaggio ameno, felice, ne segue un altro «terribile». Un «bel fanciullo coi folti capelli» appare nella sua visione; costui lo guarda serenamente, eppure Castorp è improvvisamente colto da un "pallido terrore e da un vago presentimento» di morte: accanto al fanciullo stanno due donne, madre e figlia, «con le palpebre abbassate sopra gli occhi dalla pupilla spenta», vestite con delle «tuniche». Più in fondo, Castorp vede con sconcerto («le ginocchia quasi gli si piegavano per lo spavento») altre due donne "grigie, mezze nude» che fanno a brandelli un bambino sussurrando parole incomprensibili; «avrebbe voluto fuggire, ma gli sembrava di essere inchiodato al suolo».

30 Anche quello di Hans Castorp non è un vero e proprio sogno ma una sorta di dormiveglia che, subito dopo quelle visioni, fa scaturire in lui delle riflessioni (sottolineate da Bufalino nel libro in suo possesso) sulla condizione umana, «convivenza cortese, intelligente e rispettosa, dietro la quale, nel tempo, si svolge l'orribile pasto cruento» e sul significato di «grande potenza» che la morte assume nell'esistenza dell'uomo. Nonostante ciò, pensa Castorp, «tutto l'interesse per la malattia e per la morte non è che un modo di esprimere l'interesse per la vita». Castorp perviene attraverso il sogno al senso più profondo della sua esperienza nel sanatorio, così come il protagonista di Diceria dell'untore, una volta scampato alla morte, come il 'fratello' tedesco, comprende quel sogno premonitore che nella sua descrizione rimanda altresì alla visione di Gustav von Aschenbach della Morte a Venezia:

Sapevo quel che m'aspettava, un consesso che sconosciuto non m'era. Di fantasmi grigi, di ombre vestite di tuniche bianche come impermeabili bianchi, non so se uomini o donne, ma no, erano uomini e donne insieme, una giuria di milioni sulle soglie di una fossa senza fondo. E m'incolpavano, mi scolpavano, mi gridavano con spente orbite: vattene via, sàlvati almeno tu. Riconobbi allora, o credetti, l'ingranaggio del mio desiderio diviso e del mio sogno di tutte le notti ${ }^{37}$.

31 Nel segnalare le pagine relative alla sezione Neve nei suoi appunti, Bufalino sembra condividere le riflessioni scaturite dalle visioni di Castorp e il significato recondito, svelato proprio in quelle pagine, del romanzo di Mann: il ribaltamento della concezione 
della morte in amore per la vita, infatti, suggerisce una chiave di lettura nuova di Diceria dell'untore. Se il romanzo racconta l'esistenza degli amici morti a Reggio o a Scandiano - secondo l'interpretazione resa possibile dai nuovi dati ricavabili dagli autografi ${ }^{38}$-, la preponderanza della tematica della morte, pervasiva nella sua opera, può essere intesa anche come celebrazione della vita di tutti coloro che Bufalino vide morire negli anni trascorsi in Emilia, funzione in parte assolta dal «miracolo del Bis» che la scrittura concede ai personaggi e, quindi, a quelle persone.

Nel racconto di Kafka si narra invece, come indica il titolo, la visione onirica di K. che, passeggiando per una strada di campagna e attraversando "sentieri artificiosamente disposti, inutilmente tortuosi», sui quali «[scivola] come mantenendosi su di una rapida corrente, stabilmente sospeso per aria $»^{39}$, finisce in un cimitero nel quale vede dei tumuli scavati di fresco. K. prova una sorta di attrazione a cui tenta di sottrarsi. Eppure, dopo un'inutile corsa, vacilla «cadendo poi in ginocchio proprio dinanzi al tumulo»; lì vi sono due uomini che tengono tra le mani una lapide sulla quale un artista è intento a scrivere il nome del defunto. Dopo un'iniziale titubanza, l'artista comincia a scrivere le iniziali di K. e la cavità, dalle profonde "pareti scoscese», improvvisamente si apre facendo sprofondare il protagonista, trasportato come «da una dolce corrente».

In questo racconto sono presenti gli stessi elementi narrativi che abbiamo incontrato nel sesto capitolo della Montagna incantata: la passeggiata, la presenza di uomini che adombrano un destino di morte, la discesa sospinta dal vento, l'incursione nelle profondità di una 'grotta'. Bufalino sembra riprendere sia il significato sotteso al brano di Mann, sia la suggestione figurativa presente in entrambi i testi di Mann e di Kafka. Ma si può supporre che per Bufalino fossero più importanti le descrizioni fortemente artistiche e simboliche, che i temi filosofici cui i testi alludono o la ripresa della mitologia greca nel caso della sequenza del sogno di Aschenbach.

Se la critica concorda nell'affermare che la trama di Diceria dell'untore richiama quella della Montagna incantata - per l'ambientazione nel sanatorio e per la guarigione di entrambi i protagonisti dei due romanzi, cui aggiungerei il rapporto tra il giovane Hans Castorp e il filosofo Settembrini, che ricalca quello tra il protagonista e il Gran Magro -, questa lista di opere ci consente ora di circoscrivere questi 'prestiti' ad alcuni precisi segmenti narrativi che da un lato offrono nuove chiavi interpretative per leggere alcuni passi dell'opera bufaliniana, dall'altro sembrano confermare almeno in parte quanto lo scrittore dichiarò, ovvero di non essersi ispirato per il romanzo all'opera di Mann: «Non è la Montagna incantata che mi ha ispirato. L'ho letta nel '43, non ero ancora ammalato, non ho sentito allora una consonanza di temi. Il Mann che mi è più vicino è quello di Morte a Venezia, e certe immagini del Doktor Faustus, mentre escludo nel modo più totale una derivazione tra la Montagna e la Diceria» ${ }^{40}$. Bufalino non mentiva. Gli esempi analizzati dimostrano che il nesso intertestuale tra Diceria dell'untore e La morte a Venezia è più esplicito e pervasivo di quello con la Montagna incantata. La rielaborazione bufaliniana della materia narrata nella sequenza Neve, infatti, non sembra sufficiente a considerare La montagna incantata come il modello narrativo di riferimento del capolavoro bufaliniano. Quella di Bufalino sembra piuttosto un'immedesimazione con il protagonista a causa della malattia, un rispecchiamento biografico più che letterario.

La presenza di questa lista tra il materiale redazionale delle Istruzioni per l'uso è dunque importante non soltanto perché permette di correggere attraverso nuove piste ermeneutiche il capolavoro d'esordio, che potrebbero essere ulteriormente 
approfondite attraverso un'analisi complessiva dei testi citati, ma anche perché consente di ricostruire il processo documentario condotto da Bufalino per la stesura del romanzo. Come abbiamo accennato all'inizio, le modalità di rielaborazione delle letture e delle fonti possono essere schematizzate in due pratiche differenti: quella tematica e quella citatoria. La prima appartiene al Bufalino antologista che procede raggruppando le opere per nuclei tematici individuando alcuni modelli, o 'suggestioni immaginative' riconducibili alla lettura e all'appropriazione dei testi, con i quali confrontarsi per descrivere gli ambienti, i personaggi (come la pioggia, il sanatorio, il personaggio di Marta); la seconda, invece, appartiene allo scrittore che ricerca un confronto diretto, attraverso un'intertestualità più esplicita, con gli autori per lui rappresentativi di una tradizione letteraria, come Kafka e Mann, che lui sente affini non soltanto per il tema trattato, ma soprattutto per il loro coinvolgimento personale e biografico nella vicenda narrata, ovvero la malattia come nel caso di Kafka, intesa non soltanto come percorso individuale (Castorp nella Montagna incantata), ma anche collettivo (il colera della Morte a Venezia).

L'analisi di questi appunti, se da un lato ci permette di arricchire il corpus dei riferimenti pubblicati da Bufalino nelle Istruzioni per l'uso, dall'altro sembra confermare l'originalità della sua scrittura, poliedrica e stratificata: l'ispirazione tratta dai grandi capolavori della letteratura moderna e contemporanea si configura, infatti, come il naturale esito di una profonda quanto vasta cultura (e sensibilità letteraria), maturata nel tempo ed esercitata lungamente nella pratica della lettura.

Sebbene Bufalino avesse taciuto le altre (importanti) fonti di Diceria dell'untore, la presenza di questo documento, rimasto sino a oggi inedito, tra le carte da lui donate alla Fondazione di Comiso, nonché la corrispondenza tra i testi menzionati nella lista e le opere conservate nella biblioteca personale, lascia supporre una precisa volontà d'autore, ovvero quella di consentire lo svelamento di alcune chiavi interpretative al suo capolavoro soltanto dopo la morte. Come ci si aspetterebbe da un abile scrittore di gialli, Bufalino ha disseminato di indizi il suo archivio, lasciando ai futuri lettori il compito di rintracciarli e collocarli all'interno di un sistema letterario potenziale.

\section{NOTE}

1. Gli esemplari portano la data dell'11 febbraio 1981.

2. G. Bufalino, Istruzioni per l'uso, in Id., Opere 1981.1988, a cura di M. Corti, F. Caputo, Milano, Bompiani, 1992, p. 1297.

3. Si tratta di un foglio di computisteria manoscritto a penna nera sul recto e sul verso con correzioni a penna rossa e numerato 55 sul recto dall'autore, cfr. «Fondazione Gesualdo Bufalino» di Comiso (d'ora innanzi Comiso FB), FSGB (01), c. $55 r$ nda. Che questa carta si riferisca alla redazione di Diceria dell'untore è confermato dalle epigrafi riportate nel margine inferiore (da Shakespeare a Tucidide). In essa sono trascritti, inoltre, gli allegati previsti come il «Diario alla Rocca», qui chiamato «Diario di lavoro», dal quale desumere gli «intercalari e i corsivi». Il foglio fa parte di un fascicolo di $21 \mathrm{cc}$. sciolte, ordinate dall'autore, corrispondenti rispettivamente a: appunti per la redazione del capitolo Giunte e allegati dell'ultima stesura di Diceria dell'untore 
classificata G e conservata presso il «Centro Manoscritti» dell'Università di Pavia (cc. da 1 a 3); materiali preparatori dell'edizione non venale delle Istruzioni per l'uso (cc. da 4 a 11); appunti per la traduzione in spagnolo delle Istruzioni, successivi alla loro uscita, in abbinamento al romanzo per il Club degli editori nel 1982 (cc. 12-14); elaborazione delle Istruzioni per la prima edizione tascabile Bompiani uscita nel 1992 che prevedeva, tra l'altro, anche il capitolo Passatempi alla Rocca di Calende greche, romanzo pubblicato nello stesso 1992 (cc.15-21). Per la funzione del «Diario alla Rocca» nell'economia narrativa e strutturale di Diceria dell'untore, mi permetto di rimandare a G. Cacciatore, L'«Opus perpetuum» di Gesualdo Bufalino, in N. Zago e G. Traina (a cura di), Il miglior fabbro. Bufalino tra tradizione e sperimentazione, Leonforte, Euno Edizioni, 2014, pp. 173-204.

4. Comiso FB, FSGB, c. $55 r$ nda. Esprimo il mio sentito ringraziamento al Direttore scientifico della «Fondazione Gesualdo Bufalino», il prof. Nunzio Zago per avermi accordato l'accesso al prezioso materiale autografo ivi conservato, e al bibliotecario Giovanni Iemulo per aver accompagnato le mie ricerche con professionalità e generosità.

5. Mi riferisco, in particolare, agli studi di Claudia Carmina e di Stefano Lazzarin pubblicati nel presente numero, a quello di Giuseppe Traina (Presenze linguistiche e tematiche della poesia montaliana in «Diceria dell'untore» di Gesualdo Bufalino, «Siculorum Gymnasium», n. s., a. XLII, $\mathrm{n}^{\mathrm{i}}$ 1-2, gennaio-dicembre 1990, pp. 239-271) e di Vania Sebben (La citazione in «Diceria dell'untore» di Bufalino, in Piccole finzioni con importanza, a cura di N. Roelens e I. Lanslots, Ravenna, Longo, 1993, pp. 167-170). Per uno studio sull'opera si vedano le due monografie di N. Zago, Gesualdo Bufalino. La figura e l'opera, Marina di Patti, Il Pungitopo, 1987 e la recente I sortilegi della parola. Studi su Gesualdo Bufalino, Leonforte, Euno Edizioni, 2016. Si veda anche, sempre di Traina, «La felicità esiste. Ne ho sentito parlare» Gesualdo Bufalino narratore, Cuneo, Nerosubianco, 2012. In Dicerie dell'autore. Temi e forme della narrativa di Bufalino (Firenze, Olschki, 2005) Marina Paino analizza sia il rapporto tra autore, narratore e protagonista nell'opera di Bufalino, dando particolare risalto allo scarto tra la biografia e la fictio letteraria, sia i temi pervasivi della sua opera come il binomio malattia-salute, ricordo e menzogna, declinata anche nelle forme della teatralità dei «personaggi-burattini» bufaliniani, analisi poi approfondite anche nel recente La stanza degli specchi. 'Esercizi di lettura' sul romanzo di Bufalino, Acireale-Roma, Bonanno, 2015. Per un'analisi linguistica del romanzo si legga F. Caputo, Diceria dell'untore. Sondaggi sulla sintassi, in Simile a un colombo viaggiatore. Per Bufalino (a cura di N. Zago, Comiso, Salarchi Immagini, 1998) pp. 83-96; nello stesso volume, inoltre, si legga il saggio di Giuseppe Pitrolo dedicato al rapporto tra memoria e finzione, Gli inventati ricordi di Gesualdo Bufalino: l'ambiguità e la memoria in Diceria dell'untore (pp.61-72). Per una lettura critica approfondita dell'opera rimando alla sezione Un romanzo della tarda modernità: Diceria dell'untore (pp. 185-320) contenuta nel primo «Quaderno della Fondazione Bufalino», Bufalino narratore fra cinema, musica, tradizione (a cura di N. Zago, Comiso, Salarchi Immagini, 2002) si vedano i saggi di N.Zago, Nascita di un romanzo (e di un'amicizia), M. Guglielminetti, Il codice autobiografico della Diceria dell'untore, A. Di Grado, "Che mastro questo don Gesualdo!», R. M. Monastra, Diceria dell'untore tra sacro e profano, G. Traina, Un palcoscenico di voci soliste? Il gioco dei personaggi in Diceria dell'untore. Sulla religione nell'opera di Bufalino si veda A. Cinquegrani, La partita a scacchi con Dio, cit., mentre per un approfondimento dell'analisi lessicale in Diceria dell'untore si veda A. Sichera, Echi scritturali e lessico religioso nel primo Bufalino, in Il miglio fabbro. Bufalino fra tradizione e sperimentazione, a cura di N. Zago e G. Traina, cit., pp. 85-104.

6. Restano escluse da questa mappatura le opere cui Bufalino fa riferimento nella loro interezza, come Le città del mondo di Vittorini, il «Giornale di Sicilia» dell'anno 1946, i riferimenti all'Enciclopedia.

7. A eccezione della Morte a Venezia, di cui Bufalino possiede un'edizione del 1981, e Le città del mondo di Vittorini, compreso in Romanzi, Mondadori, «i Meridiani», Milano, 1982, tutte le citazioni a seguire nel testo sono desunte dalle edizioni bufaliniane: Alain-Fournier, Il grande 
amico, Milano, Mondadori, 1947; M. Soldati, Il padre degli orfani, in Id., A cena col commendatore, Milano, Mondadori, 1964; Colette, Il grano d'erba e Il mio tirocinio, Milano, Mondadori, 1964; M. Tobino, Le libere donne di Magliano, Milano, Mondadori, 1967; V. Pratolini, La costanza della ragione, Milano, Mondadori, 1974; F. Kafka, Il sogno, in Id., I racconti, Milano, Longanesi, 1955; P. Nizan, La cospirazione, Milano, Mondadori, 1961; J. Conrad, Lo strale d'oro, Milano, Bietti, 1934; T. Mann, La montagna incantata, Milano, Dall'Oglio, 1945; T. Landolfi, Des mois, Milano, Longanesi, 1972.

8. I tempi di stesura di Diceria dell'untore sono chiariti dallo stesso Bufalino nell'intervista concesso all'amico Leonardo Sciascia nel 1981: «L'ho pensato e abbozzato verso il '50, l'ho scritto nel '71. Da allora una revisione ininterrotta: fino alle bozze di stampa» (L. Sciascia, Che mastro questo don Gesualdo!, «L'Espresso», 1 marzo 1981).

9. Per un ragguaglio sulle antologie inedite si legga G. Traina, L'ingegnere di Babele. Bufalino antologista, in Il miglior fabbro, cit., pp. 125-150.

10. G. Bufalino, Diceria dell'untore, in Id., Opere 1981.1988, cit., pp. 132-133.

11. Cfr. M. Tobino, Le libere donne di Magliano, cit., p. 102.

12. Cfr. T. Landolfi, Des mois, cit., p. 123.

13. «Troppo netto si staccava l'azzurro sui doccioni della Rocca, con un solo falchetto lassù, e nessuno scudo di nuvola che stornasse l'avvento del giorno di Dio. Poiché c'è un giorno, uno solo, di luglio, nell'isola, che si snatura dagli altri e non si dimentica più» (G. Bufalino, Diceria dell'untore, cit., p. 53).

14. «[...] il manicomio senza la distrazione dell'estate, torna evidente, vivo, mio dominatore [...] in un'aria celeste e rarefatta ma nella quale si sentono bene le parole» ( $\mathrm{Cfr}$. M. Tobino, Le libere donne di Magliano, cit., p. 134).

15. G. Bufalino, Diceria dell'untore, cit., p. 79.

16. Alain-Fournier, Il grande amico, cit., p. 60.

17. G. Bufalino, Diceria dell'untore, cit., p. 23.

18. «[...] si coricava di fianco alla bandiera del reggimento e a casse piene di trofei di guerra, elmetti, bandiere tedesche, galloni strappati dalle maniche dei morti, di fronte a una finestra lacerata dai lampi violetti di Parigi, ascoltando, quando non dormiva, fischiare i treni del Nord. Pensava solo a scappare» (P. Nizan, La cospirazione, cit., p. 88).

19. «[...] davanti al casello dove abitavo, mentre aspettavo i treni, la notte. [...] Avrei voluto partire un mattino su uno di quei treni, alla ventura» (G. Bufalino, Diceria dell'untore, cit., p. 66).

20. Ivi, p. 28.

21. Cfr. P. Nizan, La cospirazione, cit., p. 51.

22. Cfr. M. Soldati, Il padre degli orfani, cit., p. 138.

23. Cfr. J. Conrad, Lo strale d'oro, cit., p. 101.

24. M. Tobino, Le libere donne di Magliano, cit., p. 132.

25. Ivi, p. 18.

26. Ivi, p. 118.

27. V. Pratolini, La costanza della ragione, cit., p. 287.

28. Cfr. T. Mann, La morte a Venezia, in Id., Romanzi brevi, a cura di Roberto Fertonani, Milano, Arnoldo Mondadori Editore, 1977, pp. 198-227.

29. «Mentre, sopra ogni altra cosa, soverchiando ogni lutto o splendore della memoria, ferinamente trionfava nel vento il tanfo della città bombardata, della sua bocca nera, del turpe esibito suo pube d'uccisa. Lo stesso tanfo che sentivamo ora salire dai nostri guanciali, un'altra guerra ci toccava combattere contro Goti più solerti e feroci» (Diceria dell'untore, cit., p. 28).

30. «La Viola [...] sembra una libellula. Magra, spiritata, agile. [...] È in una solitudine che lei non sente affatto. (Le donne magre con l'andare degli anni mantengono di più il respiro giovanile)» (M. Tobino, Libere donne di Magliano, cit., p. 18). 
31. In Colette, Il grano in erba, cit., p. 190, si legge: «Un abito per bambola di carta a piccoli volanti in gradazione, di taffetà cangiante blu e verde... un abito color pelle di Polaire con un diadema da pellerossa viola... un abito da negra, bianco neve... un abito che aveva sotto la gonna l'arcobaleno spiegato intorno a due gambe calzate di sera, sottili, nere, dure, di un'eleganza esotica...».

32. «E come mi parve bella nell'atto di balzare dal predellino del tram e di incamminarsi verso di me con passo eccitato, facendo svolazzare la gonna in movenze di mulinello attorno alle lievi elegantissime gambe. Mi schiaffeggiò affettuosamente col guanto di pizzo, con l'altra mano reggeva un'ampia borsa, colma di cianfrusaglie da donna. Persino un occhiale da sole ne estrasse, e una lunga sciarpa di seta» (Diceria dell'untore, p. 112).

33. M. Tobino, Le libere donne di Magliano, cit., p. 134.

34. G. Bufalino, Diceria dell'untore, cit., p. 135.

35. «O quando tutte le notti, per pigrizia, per avarizia, tornavo a sognare lo stesso sogno: una strada color cenere, piatta, che scorre con andamento di fiume fra due muri più alti della statura di uomo; poi si rompe, strapiomba sul vuoto» (ivi, p. 9).

36. Si cita diffusamente dall'edizione della Montagna incantata posseduta da Bufalino e in particolare dalle pp. 475-505.

37. G. Bufalino, Diceria dell'untore, cit., p. 124. Per il sogno di Gustav von Aschenbach cfr. T. Mann, La morte a Venezia, cit., pp. 217-219.

38. Mi riferisco alla ricostruzione biografica degli anni della guerra e del dopoguerra, di cui ho dato alcune sommarie informazioni in G. Cacciatore, L'archivio di Bufalino: una (auto)biografia a futura memoria, consultabile all'indirizzo: <www.italianisti.it/upload/userfiles/files/CACCIATORE Giulia.pdf> (16 marzo 2019).

39. Si cita dall'edizione dei Racconti Longanesi del 1955 posseduta da Bufalino, pp. 122-124.

40. F. Santini, "La mia Sicilia è un museo d'ombre e io vivo in un buco nero", «Tuttolibri», 11 luglio 1981.

\section{RIASSUNTI}

L'articolo analizza, attraverso un confronto testuale con i libri conservati nella biblioteca personale dell'autore, alcune fonti letterarie confluite nel tessuto narrativo di Diceria dell'untore, e da lui taciute nelle Istruzioni per l'uso. Quest'analisi è resa possibile da una lista di autori (Pratolini, Landolfi, Tobino, Alain Fournier, Nizan, Colette, Conrad, Mann e Kafka) e di opere redatta da Bufalino, ma rimasta sinora inedita.

Cet article analyse, à travers une comparaison textuelle avec les livres conservés dans la bibliothèque privée de l'écrivain, certaines sources littéraires intégrées dans le tissu narratif de Diceria dell'untore (Le semeur de peste), mais qui ne sont pas indiquées dans les Istruzioni per l'uso [Mode d'emploi]. Cette analyse est rendue possible par une liste d'auteurs (Pratolini, Landolfi, Tobino, Alain Fournier, Nizan, Colette, Conrad, Mann et Kafka) et d'ouvrages rédigée par Bufalino mais restée jusqu'ici inédite.

This essay analyses, through a textual comparison with the books present in the private library of the writer, some literary sources that Bufalino incorporated in the text of Diceria dell'untore [The Plague Spreader's Tale, also translated as The Plague Sower] but didn't mention in his Directions for Use. The analysis is made possible by a list of authors (Pratolini, Landolfi, Tobino, Alain 
Fournier, Nizan, Colette, Conrad, Mann and Kafka) and books, a list drafted by Bufalino but unpublished so far.

\section{INDICE}

Mots-clés : Gesualdo Bufalino, Le semeur de peste, sources littéraires, bibliothèque d'auteur Parole chiave : Gesualdo Bufalino, Diceria dell'untore, fonti letterarie, biblioteca d'autore Keywords : Gesualdo Bufalino, The Plague Sower, literary sources, author's library

\section{AUTORE}

\section{GIULIA CACCIATORE}

Università di Catania 